\title{
Generalizing and optimizing fractional frequency reuse in broadband cellular radio access networks
}

\author{
Lei Chen ${ }^{1 *}$ and Di Yuan ${ }^{1}$
}

\begin{abstract}
For broadband cellular access based on orthogonal frequency division multiple access (OFDMA), fractional frequency reuse (FFR) is one of the key concepts for mitigating inter-cell interference and optimizing cell-edge performance. In standard FFR, the number of OFDMA sub-bands and the reuse factor are fixed. Whereas this works well for an idealized cell pattern, it is neither directly applicable nor adequate for real-life networks with irregular cell layouts. In this article, we consider a generalized FFR (GFFR) scheme to allow for flexibility in the total number of sub-bands as well as the number of sub-bands in each cell-edge zone, to enable network-adaptive FFR. In addition, the GFFR scheme takes power assignment in consideration. We formalize the complexity of the optimization problem, and develop an optimization algorithm based on local search to maximize the cell-edge throughput. Numerical results using networks with realistic radio propagation conditions demonstrate the applicability of the GFFR scheme in performance engineering of OFDMA networks.
\end{abstract}

\section{Introduction}

Orthogonal frequency division multiple access (OFDMA) is a current technology for broadband radio access. In OFDMA, the radio spectrum is split into a large number of channels, referred to as sub-carriers. Data transmission is performed simultaneously over multiple sub-carriers, each carrying a low-rate bit stream. OFDMA is very flexible in exploring multi-user diversity with high spectrum efficiency and scalability. The technique is part of the downlink air interface in the fourth generation cellular systems based on 3GPP long term evolution (LTE) standards (see [1]), and IEEE 802.16 WiMAX (see [2]).

Orthogonal frequency division multiple access subcarriers are orthogonal to each other. As a result, intracell interference is not present. Inter-cell interference, on the other hand, becomes a performance-limiting factor. For this reason, interference mitigation has become an important topic in performance engineering of OFDMA networks.

*Correspondence: Lei.Chen@liu.se

1 Department of Science and Technology, Campus Norrköping, Linköping University, SE-60174, Norrköping, Sweden

\section{Fractional frequency reuse}

One sub-carrier allocation scheme in multi-cell OFDMA networks is frequency reuse with factor one (reuse-1), in which the entire spectrum is made available in all cells. Here and throughout the article, a reuse factor of $X$ means that the spectrum block in question is used in every group of $X$ cells. Users that benefit from the reuse-1 scheme are those located close to a base station antenna, i.e., users in the cell center. Because the channel condition is good and the interference from other cells is relatively low, the throughput in cell-center zones grows by bandwidth. For users located at cell-edge areas, however, the performance typically suffers severely in the reuse- 1 scheme, because of the high interference from the surrounding cells in relation to the signal of the home cell. In other words, celledge zones are much more sensitive to interference than bandwidth. Previous studies (e.g., [3]) indicate that, if the overall system throughput is the only performance target, then reuse- 1 is the best choice. At the same time, the resulting performance in the cell-edge zones tends to be unacceptably low.

To balance the performance of cell center against that of cell edge, interference avoidance and mitigation techniques have been investigated in recent years. One of the key concepts specifically addressing the performance of

\section{Springer}

(c) 2012 Chen and Yuan; licensee Springer. This is an Open Access article distributed under the terms of the Creative Commons Attribution License (http://creativecommons.org/licenses/by/2.0), which permits unrestricted use, distribution, and reproduction in any medium, provided the original work is properly cited. 
cell edge is fractional frequency reuse (FFR), see, for example, [4-6]. In FFR, the service area of every cell is split into a center zone and an edge zone. The spectrum is correspondingly partitioned into two parts. One part is allocated with reuse- 1 in all cell-center zones. The second part is further split into sub-bands. These sub-bands, to be used in the cell-edges zones, have a higher reuse factor. As a result, significant interference reduction is achieved in cell-edge zones. In standard FFR, derived for an ideal network layout with hexagonally shaped cells, the edge band is split into three sub-bands, each with a reuse factor of three (reuse-3); every edge zone is allocated one of the three sub-bands, see Figure 1 for an illustration.

For the cell layout in Figure 1, the standard sub-band allocation pattern with reuse- 3 is very intuitive. The allocation pattern ensures that the sub-band of a cell-edge zone is not reused in any of the neighboring cells. In reallife cellular networks, however, the amount of interference is very irregular over the service area. The cells differ greatly in the number of significant interferers as well as the respective amounts of interference, causing difficulties in applying standard FFR. As the number of surrounding cells varies from one cell to another, allocating the sub-bands optimally is not straightforward. For the same reason, a single reuse factor, if applicable at all, is no longer optimal. In addition, because the sensitivity to interference varies by cell, the allocation of one sub-band per edge zone may not be adequate. Finally, scalability becomes an issue, because it is not optimal to replicate the allocation pattern of one part of the network to another.

\section{A generalized FFR scheme}

In this article we present and evaluate a generalized FFR (GFFR) scheme, in order to overcome the limitations of standard FFR in dealing with real-life networks with irregular cell layout. As a result, the allocation pattern is adapted to the characteristics of each individual network.

The GFFR scheme that we consider extends the standard one in three aspects. First, the frequency band used for cell-edge zones can be partitioned into any number of sub-bands. Doing so is potentially useful for interference avoidance in cells with many surrounding interfering

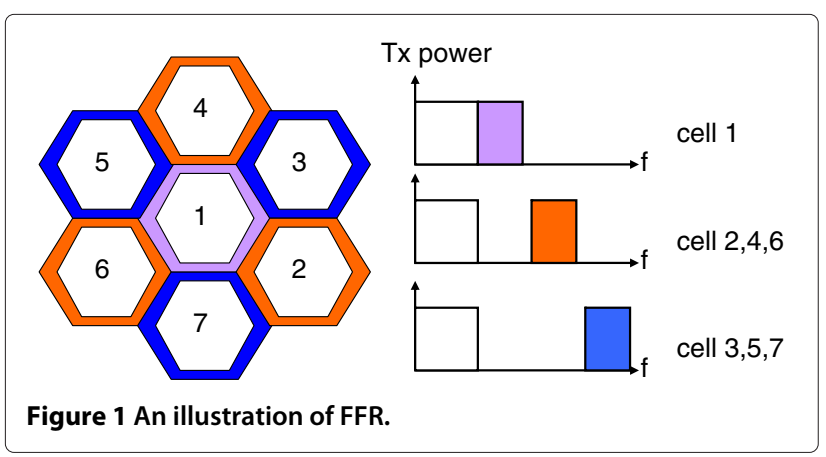

neighbors. Second, the number of sub-bands allocated to the edge zone is cell-specific. Having this flexibility is important when many sub-bands are created, since the cell-edge zones in real networks differ in their levels of sensitivity to interference respective spectrum bandwidth. Some edge zones may benefit from using few sub-bands which are not reused at all in the surrounding areas, whereas other edge zones can tolerate higher interference and consequently should be allocated more sub-bands with a higher reuse level. As the third extension of FFR, power assignment is part of the optimization framework of GFFR. In standard FFR, power is typically not a variable. The GFFR scheme allows the power to vary by cell, in order to achieve further gain in addition to that enabled by sub-band reuse optimization.

We focus on cell-edge user performance in this article. The performance metric that we use to assess sub-band allocation and power assignment targets throughput guarantee in the cell-edge zones. In the system model (see Section "The system model"), the service area is represented by a large number of pixels. For example, in one of the test network scenarios for performance evaluation (Section "Experimental results"), a pixel is a square area of size $20 \times 20 \mathrm{~m}$. For each cell-edge pixel, the performance metric is defined by the data throughput that can be guaranteed when all the interfering cells are active. In effect, this metric corresponds to the average downlink throughput with uniformly distributed cell-edge users and round-robin scheduling, and provides a throughput map over the cell-edge areas. Note that, by associating pixels with non-uniform utility parameters, the metric is easily adapted to any given user distribution with uneven traffic demand.

Figure 2 gives an illustration of GFFR, in which the granularity in creating sub-bands is high, and an edge zone may use multiple sub-bands. In addition, the power varies by cell. In the example, cell one has more edge sub-bands allocated than the other two cells, most likely because the edge zone of cell one is less interference-sensitive. At the same time, allocating more sub-bands to cell one results in higher interference to the other cells. Hence the allocation decision is based on the overall edge-zone throughput in the network. From this simple example, it is clear that allocating sub-bands and assigning power in GFFR amount to solving a combinatorial optimization problem.

Assuming that power is evenly distributed over the subbands in every cell[7-10], we consider the power assignment scheme where each cell can select, among a given set of power levels, the power to be used per sub-band. The total power used for the cell-edge zone of a cell is thus the product between the chosen power level and the number of allocated sub-bands. Thus GFFR deals with optimization in the dimension of power, in addition to spectrum. 


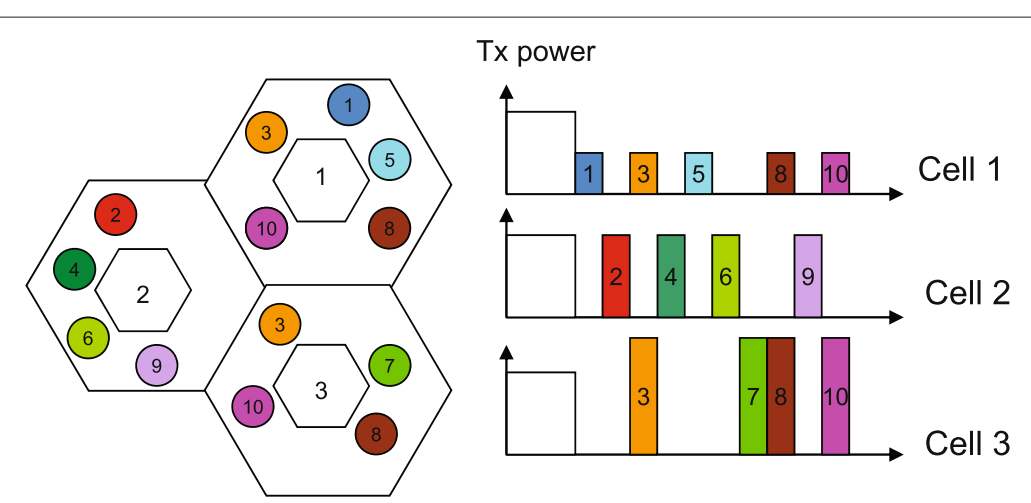

Figure 2 An illustration of the proposed GFFR scheme.

We formulate the task of sub-band allocation and power assignment as an optimization problem, and prove its complexity. A local search algorithm is developed for problem solution. The search in the algorithm is based on a neighborhood structure that finds the exact optimum of sub-band allocation and power assignment of one cell, provided that the solutions of the other cells are tentatively kept fixed. This single-cell optimization process is not straightforward, we show however that it can be implemented to run in polynomial time.

We use networks with realistic radio propagation conditions and very irregular cell layout for performance evaluation. The optimized allocation significantly goes beyond the performance of FFR with three sub-bands, which, in its turn, delivers better throughput than reuse1 in cell-edge zones. The improvement that we obtain enables better trade-off between cell-center and cell-edge performance. In addition, the information required by the algorithm's key operation can be restricted to be local to each cell. This observation forms a promising basis for developing distributed allocation algorithms.

\section{Paper outline}

The remainder of the article is organized as follows. In Section "Related works" we review related works and summarize the contributions of the current article. The system model and notation are given in Section "The system model". In Section "The optimization problem and its complexity", we formalize the optimization problem and prove its complexity. The local search algorithm is detailed in Section "Solution algorithm". We present and analyze experimental results in Section "Experimental results". Finally, Section "Conclusions" is devoted to conclusions and an outline of further research.

\section{Related works}

There is a growing amount of research on FFR and its performance evaluation. Simulations for assessing the performance of the standard FFR scheme have been conducted in [11-14]. The main conclusion is that FFR is able to improve the performance at cell-edge zones. The impact of cell-edge size on throughput has been analyzed in [15]. In [16], an analytic model for estimating the throughput of FFR is presented. The idea of using analytic models instead of simulation to assess the signal-to-interferenceand-noise ratio (SINR) for FFR is adopted also in [17]. In [18], it is shown that, if FFR is performed in a distributed fashion, and each cell selfishly optimizes the assignment of resource to its own users, the system will converge to a Nash equilibrium. Chang et al. [19] take a graph theoretical approach and formulate FFR sub-band allocation as a graph coloring problem. In [20,21], FFR sub-band allocation has been performed based on an interference graph connecting the base stations. The studies consider an ideal cell layout pattern in performance evaluation. Comparative studies of FFR and other interference-mitigating schemes, in particular the soft frequency reuse (SFR) scheme, are provided in $[22,23]$. Simulation tools and architecture support for FFR are presented in $[24,25]$. The application of FFR and its performance evaluation in femto-cell environments are examined in [26-29].

For a given set of users, the problem of allocating resource at the sub-carrier level in FFR is formulated using mathematical optimization models in [30,31]. The constraint in resource allocation is the minimum required user throughput. The solution approach is based on a relaxation of the original problem to obtain convex optimization formulations. A similar problem setting for OFDMA resource allocation is considered in [32], and solved using Lagrangian relaxation.

Extensions and variations of the basic FFR scheme have been examined in a number of recent articles. In [33], the authors propose an incremental frequency reuse (IFR) scheme to adapt resource allocation to cell load. Similar schemes that adapt sub-band allocation to traffic and user distributions are proposed in $[34,35]$. Luo et al. [36] propose algorithms to improve the throughput of best-effort traffic in OFDMA systems through automated formation 
of the FFR pattern. The work in [37] validates a clusteringbased FFR scheme. In [38], FFR is combined with interference suppression for improving spectral efficiency and power expenditure. Rahman and Yanikomeroglu [39] proposes to perform FFR-based interference avoidance at two levels by base station and a central controller, respectively. The latter conducts inter-cell coordination. An FFR scheme based on generating reports of the interference levels among neighboring cells is presented in [40]. The work in [41] augments FFR by enabling adaptive spectral sharing, and proposes a graph-theoretical solution approach. In [8], Ali and Leung presents a two-level FFR scheme. At the RNC level, groups of sub-carriers are allocated to cells. Resource allocation to individual users is then performed opportunistically by base stations. In [42], Tara and Hakima proposes an FFR-based frequency planning scheme utilizing zone switching diversity for multi-cell mobile WiMAX networks.

The current article presents significant extensions to our preliminary studies of optimal FFR in large-scale networks with irregular cell layout $[23,43]$. The works reported in the two references have several limitations. First, no theoretical result in problem complexity is provided. Second, resource allocation of every edge zone is restricted to one single sub-band. Third, uniform power is assumed in all cells. Fourth, the impact of the SINR threshold used to define cell edge is not considered. In the current article, we provide a formal proof of problem complexity and present a new optimization algorithm that overcomes the performancelimiting assumptions in $[23,43]$. In addition, we study how the cell-edge SINR threshold influences the performance.

A problem domain related to optimal FFR is frequency assignment in second generation cellular networks. In frequency assignment, the number of frequencies to be allocated to each cell is given. Typically, the objective is to minimize the total number of frequencies or to minimize the interference for a fixed set of available frequencies. For frequency assignment, we refer to the extensive surveys in $[44,45]$ and the references therein, and $[46,47]$ for generalizations of frequency assignment to frequency-hopping networks. In Section "The optimization problem and its complexity" we outline the structural differences between frequency assignment and GFFR.

Another related topic consists in the practical implementation of FFR in OFDMA-based systems, such as LTE networks. Static and semi-static FFR implementations have been discussed in $[4,6,25]$, and dynamic FFR implementations have been investigated in $[8,26,41]$. By the flexibility of GFFR, it admits to be implemented both statically or dynamically. For example, GFFR can be implemented at the top level (e.g., the RNC level in the architecture proposed in [8]) in a hierarchical resource management framework, with opportunistic resource allocation in the lower level.

Depending on the way of implementation (centralized, distributed, cluster based, etc.), information gathering and exchange are necessary at one or more levels in a network. The information required by GFFR does not differ from that in the previously proposed FFR implementations. For example, in a centralized scheduling scheme, such as the one at the top level in [8], the scheduler needs user channel state information to coordinate the resource allocation among cells. The same type of information would be gathered for GFFR. When implemented in a distributed manner, GFFR is able to, as was mentioned earlier, base its decisions on local information, provided that the neighboring cells coordinate the sequence of decision making. In such a case, the resource allocation status needs to be exchanged between eNodeBs. In LTE, this can be done through the standard X2 interface. To summarize, GFFR is a more generalized and enhanced version of FFR, and can be implemented as an integral part of the overall resource allocation scheme.

\section{The system model}

In this section, we first present the basic elements of the system model, along with introducing notation. Next, the power assignment scheme is discussed. We then present cell-edge throughput calculation for any given sub-band allocation and power assignment.

\section{Preliminaries}

We use $\mathcal{C}=\{1, \ldots, C\}$ to denote the set of cells of an OFDMA network. The service area is represented by a regular grid of a large number of pixels $\mathcal{J}=\{1, \ldots, J\}$. Each pixel $j \in \mathcal{J}$ is a small square area within which radio propagation is considered uniform. We use $g_{i j}$ to denote the total gain between the cell antenna of $i$ and pixel $j$. The gain value is typically obtained by measurements and/or prediction models of signal propagation. The service area of a cell is divided into a center zone and an edge zone. The latter represents locations that are prone to interference from the surrounding cells. Denote by $\mathcal{J}_{i}^{e}$ the set of pixels forming the edge area of cell $i$. Note that, for real-life networks, the shape of cell edge is in general irregular, just like the cell layout. Moreover, it may happen that a cell does not have an edge zone. This occurs if the signal of the cell antenna is strong over the entire cell area. Merely for simplifying the notation, we assume that all cells have edge zones in our system modeling.

The total downlink transmit power of any cell antenna is denoted by $P^{\text {Tot }}$. We assume that $P^{\text {Tot }}$ does not vary by cell, again for the sake of simplifying the notation. The spectrum is partitioned into two parts to be used by the center and edge zones, respectively. We denote the 
total bandwidth and the bandwidths allocated for center and edge zones by $B, B_{c}$, and $B_{e}$, respectively, where $B=B_{c}+B_{e}$.

The cell-center band with bandwidth $B_{c}$ is allocated with reuse- 1 in all cell-center zones, which we do not discuss further in the system model. The cell-edge band is split into equal-sized sub-bands. Let $K$ denote the number of edge sub-bands, and $\mathcal{K}=\{1, \ldots, K\}$. The bandwidth of each sub-band is $B_{\text {sub }}=\frac{B_{e}}{K}$. Moreover, we use $\sigma_{j}$ to denote the thermal noise effect in pixel $j$.

In GFFR, one or multiple sub-bands in $\mathcal{K}$ is allocated to each cell-edge zone. We represent the allocation pattern by a tuple of sets

$$
\mathbf{c}=\left(\mathcal{C}_{1}, \ldots, \mathcal{C}_{k}, \ldots \mathcal{C}_{K}\right)
$$

In (1), $\mathcal{C}_{k}$ contains the subset of cells being allocated subband $k$. Clearly, each cell will appear in at least one of the set elements in c. For sub-band allocation pattern c, we use $N_{i}$ (c) to denote the number of sub-bands allocated to the edge zone of cell $i$; this equals the number of set elements in containing cell $i$.

\section{Power assignment}

We consider the common assumption of uniform power over the edge sub-bands in each cell. Under this assumption, the power used per sub-band in cell $i$ is denoted by $p_{i}$. This is an optimization variable, and its value is to be selected from a given discrete set of power levels $\left\{P^{1}, P^{2}, \ldots, P^{L}\right\}$. The power assignment is represented by the following vector.

$$
\mathbf{p}=\left(p_{1}, \ldots, p_{i}, \ldots, p_{C}\right)
$$

The number of sub-bands of an edge zone can range between one and $K$ in GFFR. As a result, the maximum value that $p_{i}$ can take depends on the number of sub-bands used in cell $i$. Without loss of generality, we assume that $P^{L}$ equals the total power available to any cell for its edge zone (i.e., this maximum can be reached on a sub-band, provided this is the only sub-band allocated in a cell), and set its value to be equal to the total cell power scaled by the edge bandwidth, i.e., $P^{L}=$ $\frac{P^{\mathrm{Tot}} B_{e}}{B}$. Power assignment has to respect the constraint that the product between $p_{i}$ and the number of sub-bands allocated in cell $i$ does not exceed $P^{L}$. Thus for sub-band allocation pattern $\mathbf{c}$, the candidate sub-band power level of cell $i$ becomes $\mathcal{L}_{i}(\mathbf{c})=$ $\left\{1,2, \ldots, L^{\prime}\right\}$, where $L^{\prime}$ is the largest integer such that $P^{L^{\prime}} N_{i}(\mathbf{c}) \leq P^{L}$.

\section{Cell-edge throughput}

For sub-band allocation $\mathbf{c}$ and power assignment $\mathbf{p}$, the SINR of pixel $j \in \mathcal{J}_{i}^{e}$ on sub-band $k$, for which $i \in \mathcal{C}_{k}$, has the following form.

$$
\operatorname{SINR}_{i j}^{k}(\mathbf{c}, \mathbf{p})=\frac{p_{i} g_{i j}}{\sum_{h \in \mathcal{C}_{k}, h \neq i} p_{h} g_{h j}+\sigma_{j}}
$$

In (3), the numerator is the received power from cell $i$. The denominator accounts for interference from cells that reuse sub-band $k$, and the noise effect $\sigma_{j}$ in pixel $j$. Thus (3) gives the SINR value that can be guaranteed when all interfering cells on sub-band $k$ are active. The SINR can then be used to estimate the available throughput within pixel $j$, by means of a throughput function, denoted by $f$. The function is monotonous and increasing in SINR. One such function is the theoretical throughput limit given by the Shannon formula below.

$$
f\left(\operatorname{SINR}_{i j}^{k}(\mathbf{c}, \mathbf{p}), j\right)=B_{\text {sub }} \log _{2}\left(1+\operatorname{SINR}_{i j}^{k}(\mathbf{c}, \mathbf{p})\right)
$$

It should be pointed out that neither our system model nor the optimization algorithm assumes the use of (4) as the throughput function $f$. In fact, any empirical function taking into account adaptive modulation and coding can be used. The total achievable throughput at $j$, provided that the home cell is $i$, equals the sum of the function values over all allocated sub-bands, i.e.,

$$
\sum_{k: i \in \mathcal{C}_{k}} f\left(\operatorname{SINR}_{i j}^{k}(\mathbf{c}, \mathbf{p}), j\right) .
$$

\section{The optimization problem and its complexity}

From the system model, the task of applying GFFR amounts to finding a sub-band allocation $\mathbf{c}$ and a power assignment $\mathbf{p}$, such that the overall throughput of the celledge areas is maximized. The optimization is subject to the conditions that each cell-edge zone is allocated at least one sub-band, and that the power used by the sub-bands together does not exceed the power limit in any cell. The optimization problem is formulated below.

$$
\begin{array}{cl}
\max _{\mathbf{c}, \mathbf{p}} & \frac{1}{\sum_{i \in \mathcal{C}}\left|\mathcal{J}_{i}^{e}\right|} \sum_{i} \sum_{j \in \mathcal{J}_{i}^{e}} \sum_{k: i \in \mathcal{C}_{k}} f\left(\operatorname{SINR}_{i j}^{k}(\mathbf{c}, \mathbf{p}), j\right) \\
\text { s.t. } & N_{i}(\mathbf{c}) \geq 1, i \in \mathcal{C} \\
& p_{i} N_{i}(\mathbf{c}) \leq P^{L}, i \in \mathcal{C}
\end{array}
$$

The objective function is monotonously nondecreasing, and concave when the Shannon formula (4) is used for throughput calculation. System (6)-(8) defines a combinatorial optimization problem. In its general form, the problem is $N P$-hard. We formalize this result below. 
Theorem 1. The sub-band allocation and power assignment problem defined by (6)-(8) is NP-hard.

Proof. The proof of the NP-hardness of problems, or, NP-completeness of its decision version, involves a polynomial-time reduction from one of the well-known NP-complete problems. For our problem, we give a polynomial-time reduction from the vertex coloring problem. Consider any instance of vertex coloring defined on graph $G=(V, E)$. Let $n=|V|$. The decision problem is to determine, for a positive integer $K>1$, whether or not there is a feasible coloring with at most $K$ colors. We assume that $n \geq 3, K \geq 3$, and $K<n$, as otherwise determining the feasibility is trivial.

First, in this paragraph, we defines the key elements in constructing an instance of the GFFR optimization problem. The set of cells $\mathcal{C}=\{1, \ldots, n\} \cup\left\{1^{\prime}, \ldots, n^{\prime}\right\}$. All cells have one single edge pixel, denoted by $j_{i}$ for cell $i$. The basic construction is illustrated in Figure 3. In the figure, the solid lines indicate the serving relation between cells and pixels. For any $i^{\prime} \in\left\{1^{\prime}, \ldots, n^{\prime}\right\}$, cell $i$ is the only potential interferer (i.e., having a positive gain value to pixel $j_{i^{\prime}}$ ). This is indicated by the dashed lines between the upper and lower parts of the figure. For any $i \in\{1, \ldots, n\}$, its neighbors in graph $G$ are the potential interferers. For example, if nodes $i$ and $h$ are neighbors in graph $G$, then $i$ is a potentially interfering cell of $j_{h}$, and $h$ is a potentially interfering cell of $j_{i}$. None of the cells $1^{\prime} \ldots, n^{\prime}$ imposes interference to others. Consider a function of type (4), with $B_{\text {sub }}=\frac{1}{2}$. Without any loss of generality, assume the function can reach value one for some SINR. There are two power levels $(L=2)$, with $P^{1}=\frac{P^{L}}{K}$. As a result, the power per sub-band has to be $P^{1}$, if a cell is allocated more than one sub-band. Note that $L$ is a constant in the proof, hence it is independent of the size of the vertex coloring problem.

The gain values $g_{i j^{\prime}}$ and $g_{i^{\prime} j_{i^{\prime}}}$, and noise effect $\sigma_{j}$ are set such that the throughput over one sub-band at pixel $j_{i^{\prime}}$ is 1.0, if the sub-band is not allocated to cell $i$ and the power

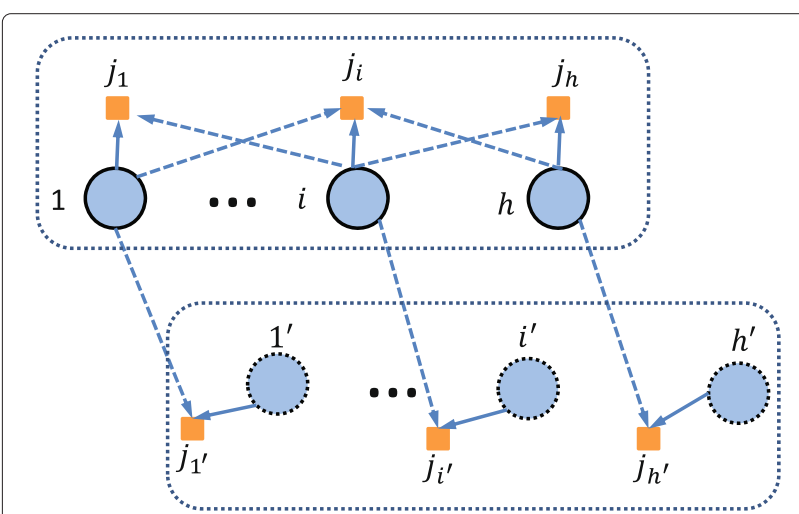

Figure 3 An illustration of the underlying idea of the proof. level in cell $i^{\prime}$ is $P^{1}$. In this case, the signal-to-noise ratio in $j_{i^{\prime}}$ is 3.0. If the sub-band is reused in $i$, the throughput of $j_{i^{\prime}}$ is $\frac{1}{2 n^{2} K}$, if the power values are $P^{L}$ and $P^{1}$ in cells $i^{\prime}$ and $i$, respectively. For cell $i$, the gain values of the potentially interfering cells (corresponding to some of the nodes in graph $G$ ) are set such that the throughput of $j_{i}$ over one sub-band is $\frac{1}{n K}$, if none of the potentially interfering cells reuses the sub-band and cell $i$ uses power level $P^{1}$. If reuse takes place, the throughput of $j_{i}$ is at most $\frac{1}{2 n K}$, even if cell $i$ uses power $P^{L}$ and there is only one interfering cell with power $P^{1}$. It can be realized that gain and noise values satisfying the above conditions can be constructed easily.

After setting the gain and noise values, we will show in this paragraph that, at optimum, any cell $i \in\{1, \ldots, n\}$ will use one single sub-band, whereas any cell $i^{\prime} \in\left\{1^{\prime}, \ldots, n^{\prime}\right\}$ will use all the $K$ sub-bands. Suppose it is optimal that cell $i$ uses multiple sub-bands (with power $P^{1}$ on each). We consider two scenarios. Assume first cell $i^{\prime}$ uses more than one sub-band (and thus also with power $P^{1}$ on each). Keeping the power fixed at $P^{1}$ and supposing that cell $i$ gives up any of the allocated sub-bands, the only cell experiencing lower throughput is $i$, and the loss is at most $\frac{1}{n K}$. The throughput increase in cell $i^{\prime}$ on the sub-band is $1-\frac{1}{2 n^{2} K}$ or 1 , depending on whether or not the sub-band is currently in use in $i^{\prime}$. Since $1-\frac{1}{2 n^{2} K}=\frac{n K-\frac{1}{2 n}}{n K}>\frac{1}{n K}$, the sub-band removal operation leads to better overall performance, contradicting the assumption of optimality. Assume now that cell $i^{\prime}$ uses a single sub-band with power $P^{L}$ (using $P^{1}$ is not optimal, because cell $i^{\prime}$ does not generate interference to other cells, see Figure 3). Consider removing the sub-bands of $i$ except one, and let $i^{\prime}$ use all the $K-1$ interference-free sub-bands with power $P^{1}$. Before the modification, the throughput in $i$ and $i^{\prime}$ are at most $(K-1) \frac{1}{n K}$ and $\frac{1}{2} \log _{2}(1+3 K)$ (because for $i^{\prime}$ the SNR with power $P^{1}$ is 3 , and $P^{L}=K P^{1}$ ), respectively. Disregarding the throughput in cell $i$ or potential performance improvement in any other cell, the new throughput of $i^{\prime}$ alone due to the modification is at least $K-1$. Comparing $K-1$ to $(K-1) \frac{1}{n K}+\frac{1}{2} \log _{2}(1+3 K)$, the former is greater for any $K \geq 3$, contradicting the optimality assumption. Therefore cell $i$ uses exactly one sub-band at optimum.

In this and the next paragraph, we will conclude the proof through the connection between the constructed vertex coloring instance and our problem. Observe that the optimum choice of any cell $i^{\prime} \in\left\{1, \ldots, n^{\prime}\right\}$ is one of the two options: To use power $P^{1}$ on all the $K$ sub-bands, among which $K-1$ are interferencefree, or power $P^{L}$ on one single sub-band. The former gives a throughput of at least $K-1$, whereas the latter gives no more than $\frac{1}{2} \log _{2}(1+3 K)$, which is smaller than $K-1$ for any $K \geq 3$. In conclusion, 
using all the $K$ sub-bands is optimal in cell $i^{\prime}$. Consequently, the throughput of $i^{\prime}$ at optimum is at least $K-1$, and strictly below $K-1+\frac{1}{2 n^{2} K}$ (because the additional term can be reached only with power $P^{L}$ ). The total throughput of $\left\{1^{\prime}, \ldots, n^{\prime}\right\}$ is in the interval $\left[n(K-1), n(K-1)+\frac{1}{2 n K}\right)$.

For cells $1, \ldots, n$, the total throughput reaches $\frac{1}{K}$, if there is a feasible coloring in $G$ with no more than $K$ colors. In this case, the overall throughput in the entire network is at least $n(K-1)+\frac{1}{K}$. If conflict in coloring has to take place between some nodes in $G$, the overall throughput of our problem instance is strictly less than $n(K-1)+\frac{1}{2 n K}+\frac{n-1}{n K}+$ $\frac{1}{2 n K}=n(K-1)+\frac{1}{K}$. Thus the feasibility of the vertex coloring instance is equivalent to whether or not the overall throughput of our problem instance reaches $n(K-1)+\frac{1}{K}$. In addition, the reduction is clearly polynomial. Hence the decision problem of (6)-(8) is NP-complete, and the optimization problem itself is NP-hard.

To some extent, optimizing sub-band allocation in GFFR is similar to the frequency assignment problem (FAP) in second generation cellular networks. There are several versions of FAP, see [45]. The version that resembles most sub-band allocation in GFFR is minimuminterference (MI) FAP. Although both problems amount to allocating frequencies (or frequency bands) to cells, there are several structural aspects in which they differ significantly. First, the number of frequencies to be allocated to each cell is part of the input of FAP, whereas for GFFR it is a decision variable. Second, MI-FAP addresses interference between pairs of cells. In our case, in contrast, cell-edge throughput is in focus, and thus the pixels forming the service area are modeled explicitly. The third difference lies in the non-linearity in the objective function (6). Due to these differences, solution algorithms developed for FAP do not apply to sub-band allocation in GFFR.

\section{Solution algorithm}

In view of the problem complexity of (6)-(8), and the objective of applying GFFR to large-scale networks, we consider a local search algorithm aimed at finding highquality solutions time-efficiently. A local search algorithm iteratively seeks solution improvement by repeatedly introducing modifications to the current solution and evaluating the outcome. The search strategy is defined by the operation used in making trial modifications. Solutions generated by the modification operation are considered neighbors to the current one, and the definition of the modification operation is also known as the neighborhood structure. The algorithm stops when no improvement can be obtained by solution modification, that is, the current solution is locally optimal in respect of its neighborhood. In this section, we present the design of the local search algorithm, in particular the neighborhood structure and how to time-efficiently evaluate the solutions in the neighborhood.

\section{Initial solution}

To obtain a starting solution, we apply a type of greedy algorithm that allocates one single sub-band to each cell. The algorithm goes through all cells $1, \ldots, C$ one by one. For each cell, the total throughput over the cell-edge pixels is computed for each of the sub-bands in $\{1, \ldots, K\}$ with the same power as reuse-1, and the sub-band leading to the highest throughput value is chosen. Once allocated a sub-band, the allocation for the cell in the initial solution is fixed when considering the remaining cells. In effect, the algorithm tends to select the sub-band for which the cell under consideration and the cells having the sub-band allocated have least interference to each other in the edge areas.

The amount of calculations in generating the initial solution is polynomial in $C$ and $K$. Note that, with an ideal hexagonal cell layout, the starting solution will coincide with standard FFR if $K=3$.

\section{Search strategy}

For (6)-(8), we will show that computing the exact optimum of sub-band allocation and power assignment of one cell can be performed in polynomial time, provided that the solutions of the remaining cells are tentatively kept fixed. Based on this result, we define the neighborhood structure as the set of alternative solutions obtained by optimizing the sub-band allocation and power assignment of one cell at a time. Among the neighboring solutions, the one having largest overall improvement is selected, and replaces the current solution. The search is repeated until the current allocation is locally optimal in every cell, i.e., no improvement can be reached by changing the allocation of any single cell.

Let the current solution be $\mathbf{c}=\left(\mathcal{C}_{1}, \ldots, \mathcal{C}_{k}, \ldots \mathcal{C}_{K}\right)$ and $\mathbf{p}=\left(p_{1}, \ldots, p_{i}, \ldots, p_{C}\right)$. The task is to find the new, optimal sub-band allocation and power assignment for each cell $i \in \mathcal{C}$, assuming that in the computation for cell $i$, the solutions of the other cells remain those specified by c and p. Suppose, for a moment, that power assignment is independent from sub-band allocation. Then changing the allocation of one sub-band does not have any impact on the throughput over the other sub-bands. If a sub-band is added to cell $i$ with a positive power level, the throughput of cell $i$ grows, and that of the cells currently reusing the sub-band will go down. The net effect can be calculated without considering the allocation of the remaining sub-bands in cell $i$. The same holds when a currently allocated sub-band changes power or is deleted completely from cell $i$. 
Consider now the connection between sub-band allocation and power assignment. Changing the number of sub-bands in a cell may enlarge or shrink the set of candidate levels in power assignment. Hence the computation does not decompose in the same way as above. As there are $\sum_{m=1}^{K}\left(\begin{array}{l}K \\ m\end{array}\right) L_{m}$ potential allocation patterns, where $L_{m}$ denotes the number of candidate power levels for $m$ sub-bands, the amount of computation is seemingly exponential in $K$. We will show, however, that determining the global optimum can be implemented to run in polynomial time.

The idea is to, for each cell $i$, go through the candidate numbers of sub-bands, and, for each $m$ between 1 and $K$, power levels $1, \ldots, L_{m}$. The overall number of combinations is polynomial in $K$ and $L$. For each of these combinations, the computational task boils down to finding, for the given power level, the $m$ best cell-edge sub-bands for cell $i$. Toward this end, all sub-bands presently used in cell $i$ are removed first, so the cell's sub-band allocation becomes empty. The throughput in cell $i$ becomes zero, and the throughput of the remaining cells that use these sub-bands are updated. Next, for each sub-band $k \in\{1, \ldots, K\}$, the throughput of cell $i$ on sub-band $k$ and the throughput loss in the other cells using this sub-band are computed. The values are put together as the performance value of sub-band $k$. Note that this computation is not dependent on the allocation nor the throughput values of the other sub-bands. Once the computation is complete for all subbands in $\{1, \ldots, K\}$, the $m$ sub-bands giving the highest performance values form the optimum, provided that the number of sub-bands allocated to cell $i$ is restricted to be $m$. Repeating the procedure for $m=1, \ldots, K$ results in the global optimum of cell $i$, assuming that the allocations in the other cells are unchanged.

The algorithm's key steps can be summarized as follows.

- Step 1: use current solution as the starting solution and, for each cell, find the best number of sub-bands, together with the power allocation, provided that the solutions for the remaining cells are tentatively kept fixed.

- Step 2: calculate the throughput change for the current cell.

- Step 3: repeat Steps 1-2 for all cells.

- Step 4: find the largest throughput improvement among all cells.

- Step 5: if the throughput improvement in Step 4 is positive, use the corresponding solution to replace the starting solution. Go back to Step 1.

A formal description of the algorithm is provided in Algorithm 1. The initial solution consists in sub-band allocation $\mathbf{c}$ and power assignment $\mathbf{p}$. These are updated by local search and returned by the algorithm as the output.
For each cell considered in the main loop, the locally optimal solution $\left(\mathbf{p}^{*}, \mathbf{c}^{*}\right)$ is initialized using $\mathbf{p}$ and $\mathbf{c}$, in lines 4-5. Thus the computations for the cells all start with the same solution. Then, sub-band removal in a cell is done in lines 6-11, resulting in a new temporary initial solution $\overline{\mathbf{c}}$. The corresponding throughput of each sub-band in all cells using the sub-band is re-calculated and updated in line 10. The for-loop spanning lines 1236 considers the number of sub-bands to be allocated in the cell under consideration, and forms the bulk of the computation. For each number $m$, another loop takes place over the corresponding candidate power levels (lines 13-35). For the power level under consideration, lines 1519 go through all sub-bands and calculate the throughput change over each sub-band if the sub-band is allocated to cell $i$. This results in a throughput change vector $\vec{\delta}$. Line 20 sorts the vector in descending order, resulting in a sorted vector $\vec{\delta}^{\prime}$ and the corresponding index vector I. Line 21 calculates the sum of the $m$ highest values in $\vec{\delta}^{\prime}$. If the sum improves over the currently best value $\xi^{*}$ (initialized to zero), the optimal solution of the cell is updated (lines 22-34), and the cell is allocated the first $m$ sub-bands in the sorted sequence with the power level selected (lines 25-33). Once all cells have been considered after a major iteration, $\mathbf{c}$ and $\mathbf{p}$ are replaced by the best single-celloptimized sub-band allocation $\mathbf{c}^{*}$ and power assignment $\mathbf{p}^{*}$, respectively. The algorithm stops when an iteration does not produce any improvement (i.e., $\xi^{*}=0$ ).

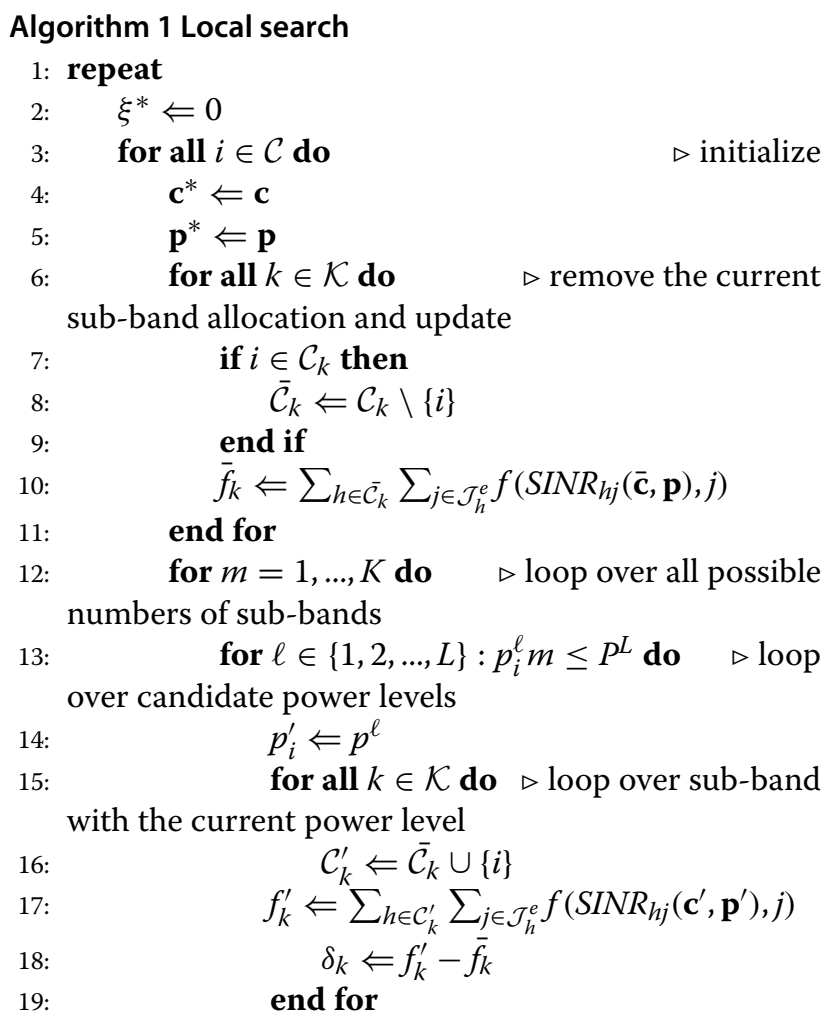




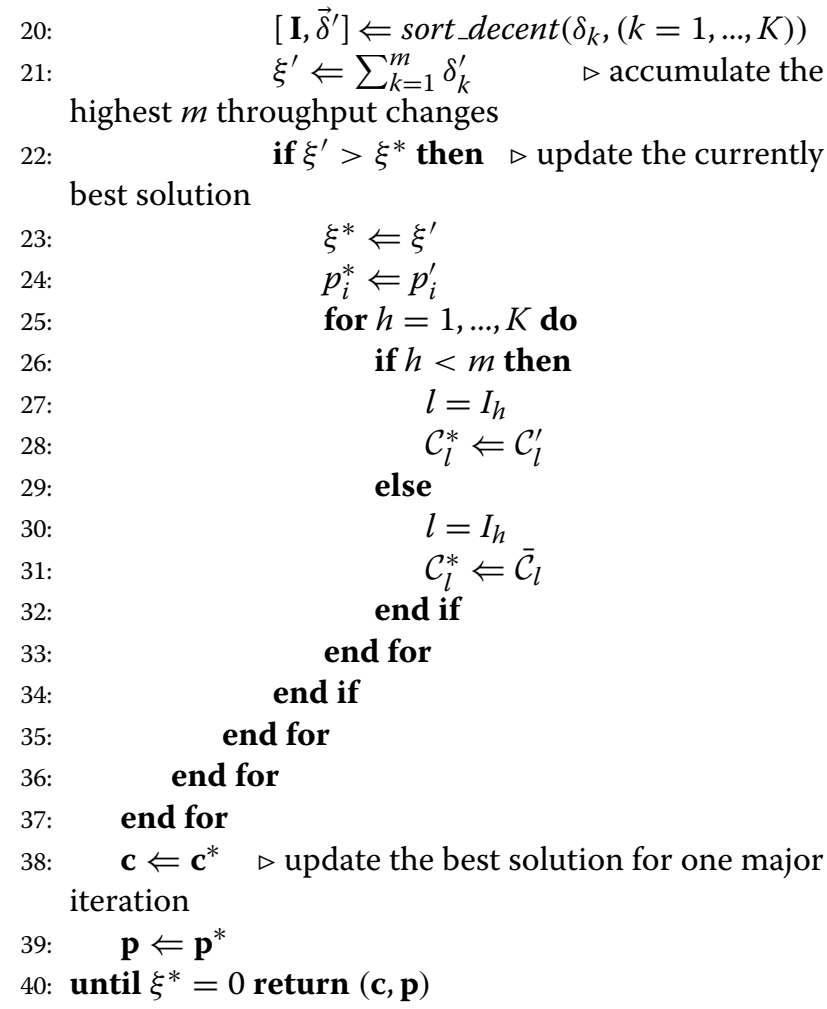

\section{Experimental results}

\section{Test networks}

We have conducted experiments using the data sets provided by the European MOMENTUM Project (see [48]). The data contains real-life cellular network deployment scenarios in several European cities. The gain values in the data sets originate from real measurements. For performance evaluation of GFFR, the test data of Berlin and Lisbon have been used. The networks correspond to large scenarios with various user densities. Figure 4 illustrates

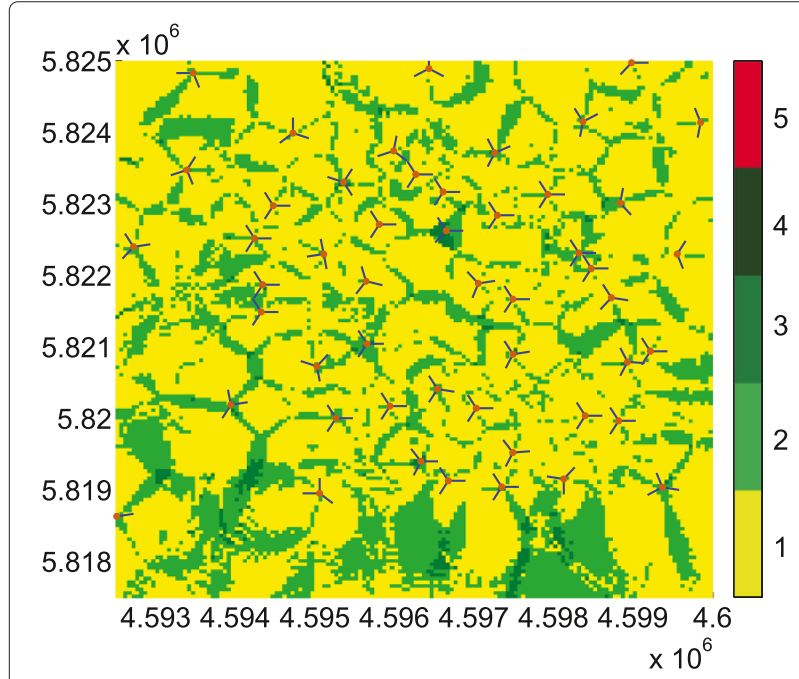

Figure 4 Cell coverage pattern of the Berlin network. the cell coverage pattern of the Berlin network. The area map consists in 22,500 pixels. The dots show base station locations, and the short lines denote the antenna directions of the cells. The color of each pixel displays the number of cells providing coverage. As can be seen from the figure, the cells differ significantly in size and shape. Moreover, the number of neighboring and hence interfering cells varies greatly in the network. For this type of scenario, a sub-band and power allocation that is optimal for a sub-group of neighboring cells will hardly perform well in another part of the network. The observation motivates the application of the GFFR scheme.

The downlink bandwidth equals $4.5 \mathrm{MHz}$ (i.e., the basic block of LTE). In the experiments, we use $B_{c}=1.8 \mathrm{MHz}$ and $B_{e}=2.7 \mathrm{MHz}$. Thus the cell-center areas lose $60 \%$ of the bandwidth in comparison to the reuse- 1 scheme. The number of sub-bands $K$ varies from 2 to 15 .

Network parameters are summarized in Table 1. Cell edge is identified using a pilot-channel SINR threshold, and the performance results depend on the threshold value. To get a good picture of the impact of cell-edge definition on performance, we use and compare four threshold values for each network, see Table 1, such that the cell-edge zones form $3,5,7$, and $10 \%$ of the total service area, respectively. For the Berlin network, the corresponding SINR threshold values are $-6.5,-5.8,-5.3$, and $-1.1 \mathrm{~dB}$. For the Lisbon network, the values are -5.0 , $-4.4,-4.0$, and $-0.8 \mathrm{~dB}$. As was discussed earlier, not all of the cells necessarily have cell-edge zone. We give the number of cells with edge zone in the table. Cell-edge throughput with reuse- 1 is also presented in the table for comparison.

In Table 1 , the cell total transmit power $P^{\text {Tot }}$ is $46 \mathrm{dBm}$, which corresponds to approximately $40 \mathrm{~W}$. Since the celledge band takes $60 \%$ of the entire bandwidth, the total power over the edge sub-bands is $P^{L}=24 \mathrm{~W}$. We require

Table 1 Network statistics and parameter setting

\begin{tabular}{lll}
\hline & Berlin & Lisbon \\
\hline Area size $\left(\mathrm{m}^{2}\right)$ & $7500 \times 7500$ & $4200 \times 5000$ \\
Number of cells & 148 & 164 \\
Area size (pixels) & 22,500 & 52,500 \\
Pixel size $\left(\mathrm{m}^{2}\right)$ & $50^{*} 50$ & $20^{*} 20$ \\
$\begin{array}{l}\text { Total DL Tx power } \\
\text { (dBm) }\end{array}$ & 46 & 46 \\
$\begin{array}{l}\text { Thermal noise (dBm) } \\
\text { Cell-edge pilot SINR }\end{array}$ & $\leq-107$ & -107 \\
threshold (dB) & $-5.3 /-1.1$ & $\leq-4.0 /-0.8$ \\
$\begin{array}{l}\text { Number of cells with } \\
\text { cell edge }\end{array}$ & $64 / 83 / 109 / 124$ & $128 / 147 / 158 / 161$ \\
$\begin{array}{l}\text { Reuse-1 cell-edge } \\
\text { throughput (Mbps) }\end{array}$ & $1.10 / 1.23 / 1.33 / 1.47$ & $1.50 / 1.66 / 1.78 / 1.93$ \\
\hline
\end{tabular}




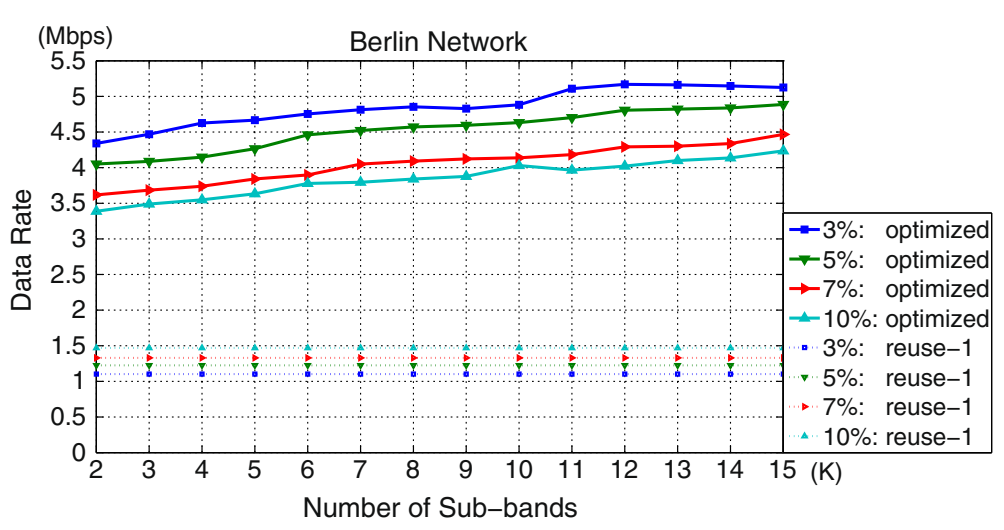

Figure 5 GFFR cell-edge performance of the Berlin network.

that all cells allocate at least $0.1 \mathrm{~W}$ to cell edge (if any). The rest of the candidate power levels are created with a step size of $0.1 \mathrm{~W}$.

The optimization algorithm is implemented in $\mathrm{C}++$ and runs on a Dell E6410 notebook with an Intel Core i7 CPU $(2.8 \mathrm{GHz})$ and $8 \mathrm{~GB}$ RAM. For each of the scenarios, we conduct 200 replications by changing the sequence of the cell during the generation of the initial solution. The results we present represent the average of the replications.

\section{Cell-edge performance}

Figures 5 and 6 display the optimized average cell-edge throughput for various values of $K$, and compare the results to reuse-1. It is clear from the figures that the commonly used reuse- 3 is not the best for an irregular cell layout. In the figures, the optimized GFFR performance tends to increase with $K$. This behavior is expected, because larger $K$ gives better granularity in resource allocation. There is however no strict monotonicity. The reason is that the solution returned by the algorithm guarantees a local optimum, which is not necessarily the global optimal solution. In the two figures, the straight lines display the throughput results of reuse-1. These results form a baseline for comparison. By definition, all sub-bands are allocated to cell edge as well as cell center in reuse-1. Therefore the performance is not dependent on $K$.

As can be seen from the figures, the optimization algorithm brings substantial throughput gain to cell edge in comparison to reuse-1. Taking the Berlin network and $5 \%$ cell edge as an example, the edge throughput grows from 1.2 to $4.5 \mathrm{Mbps}$ for small $K$, and further to $4.9 \mathrm{Mbps}$ for some of the larger $K$ values. Considering the Lisbon network and the same percentage of edge area, the throughput is $1.7 \mathrm{Mbps}$ with reuse-1, and lies between 3.4 and 4.1 Mbps for GFFR. Summarizing the results, the ranges of the improvement factor are $[2.9,4.7]$ and [2.0, $2.9]$ for the two networks, respectively, for $K=15$. Here, the improvement factor refers to the ratio between the optimized GFFR throughput and that of reuse- 1 in the cell-edge zones.

From the figures, it is apparent that the improvement decreases by the threshold value used to define the cell edge. Thus considering larger area as cell edge brings

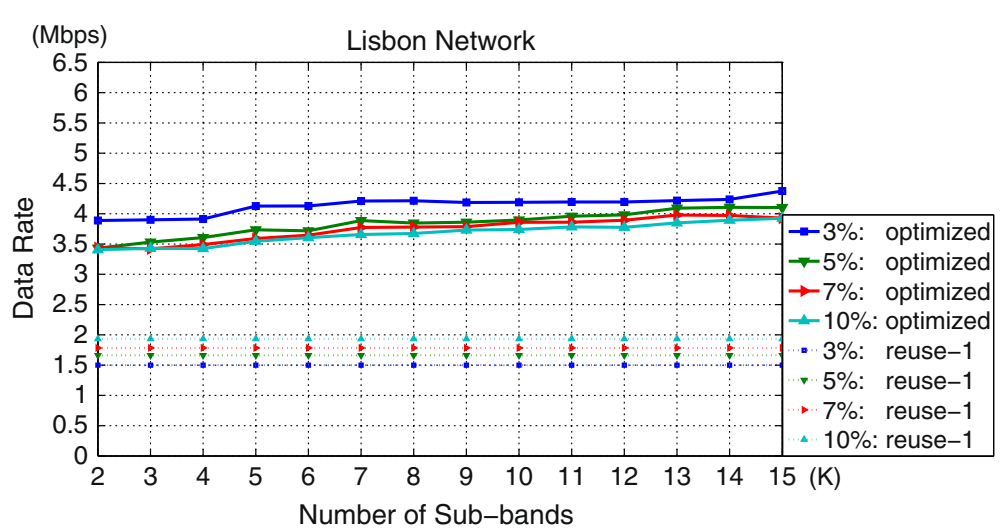

Figure 6 GFFR cell-edge performance of the Lisbon network. 


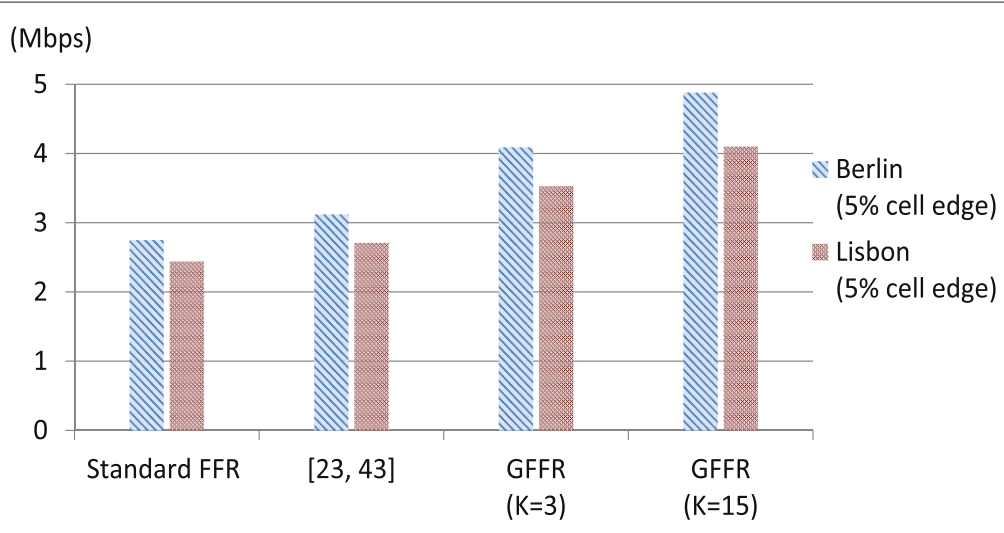

Figure 7 Performance comparison with $[23,43]$.

down the average throughput. This is because a high threshold means that larger areas are considered cell edge, although parts of these areas are bandwidth-sensitive rather than interference-sensitive. The throughput loss of these bandwidth-sensitive users contributes significantly to the decrease of the average cell-edge throughput.

Our next part of performance evaluation compares GFFR with standard FFR and the results in [23,43]. Note that standard FFR will not perform better than GFFR with $K=3$. As was discussed earlier (Section "Fractional frequency reuse"), for networks with irregular cell layout, standard FFR cannot be directly applied with a fixed frequency reuse pattern, e.g., reuse-3, because the number of neighboring cells varies greatly over the service area. Thus optimization is needed for the implementation of standard FFR for our network scenarios. Our previous works $[23,43]$ have been devoted to extending and optimizing standard FFR where the edge band can be partitioned into more than three sub-bands. Following the basic principle

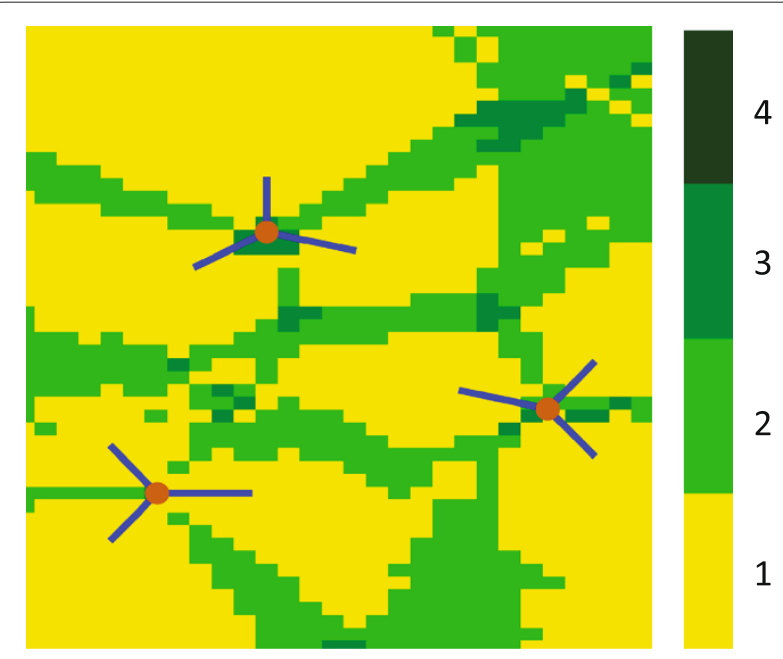

Figure 8 Illustration of the coverage pattern for a small scenario. of standard FFR, strictly one sub-band can be allocated to each of the cell-edge zones in $[23,43]$. To demonstrate the strength of GFFR, in Figure 7 we provide a comparison between GFFR, standard FFR, and our previous works in $[23,43]$ with $5 \%$ as the cell edge size.

From the figure, the improvement of GFFR over both standard FFR and $[23,43]$ is apparent. Taking the Lisbon networks as an example, standard FFR with $K=3$ gives an average throughput of $2.44 \mathrm{Mbps}$. Applying the optimization algorithm in $[23,43]$, which follows a search strategy being different from that of the current article, the average throughput result is $2.71 \mathrm{Mbps}$, giving an improvement of approximately $11 \%$ over standard FFR. GFFR has its strengths in providing the additional flexibility of power optimization as well as allowing multiple sub-bands in a cell-edge zone. For $K=3$, GFFR yields an average throughput of $3.53 \mathrm{Mbps}$, outperforming standard FFR by $45 \%$ and the results of our previous works by $30 \%$. For $K>$ 3 , additional improvement is achieved. With $K=15$, the corresponding GFFR throughput value is $4.10 \mathrm{Mbps}$, for which the relative improvement over standard FFR is $68 \%$. Similar observations can be made for the Berlin network scenario. In conclusion, the merit of GFFR in comparison to standard FFR and $[23,43]$ is significant.

For small scenarios, the global optimum may be approached by exhaustive search. This is useful for the purpose of gauging the performance of our search algorithm. Figure 8 gives an illustration of such a scenario of nine cells. For two candidate power levels and small $K$ (e.g., $K \leq 4$ ), it is computationally feasible to compute the global optimum. We have performed the computation for a number of scenarios obtained by taking small parts of the Berlin network, and compare the results to those from our local search algorithm (Section "Search strategy") for $K=3$. It turns out that the average optimality gap is less than $2 \%$. Thus, for scenarios admitting exact performance evaluation, the assessment shows close-to-optimality performance of the search algorithm. 


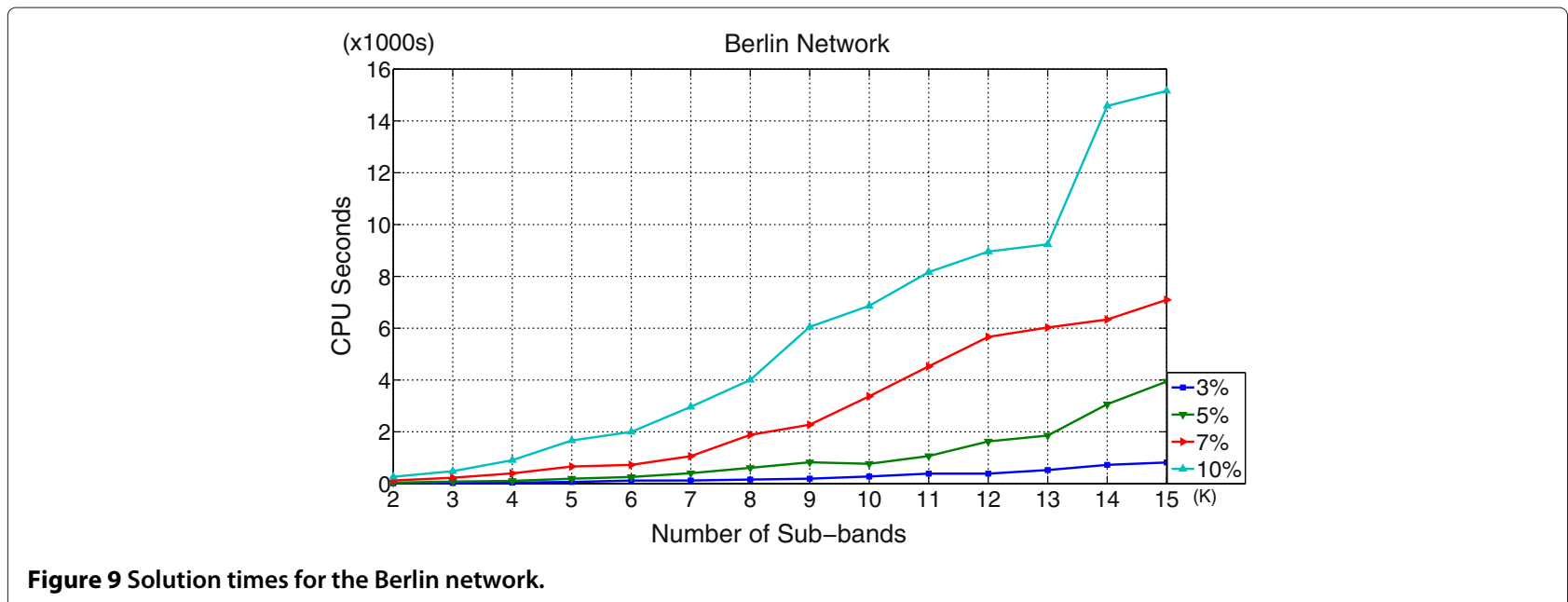

Figure 9 reports the computing times for the Berlin network. It is evident that the time grows fast in $K$ as well as in the size of the cell-edge zone. In general, increasing $K$ implies that more candidate allocation solutions must be evaluated in the search algorithm, and enlarging the edge-area size leads to a higher number of pixels and hence more computations in throughput calculation. Putting the figures of throughput and computing time together, one can observe that whereas using a higher number of $K$ makes the computing time grow rapidly, the additional performance gain is comparatively moderate. From our results, setting $K$ between 3 and 6 achieves a good balance between the performance in throughput and computational effort. In general, the choice of $K$ depends on the network characteristics and finding a proper value is not trivial. In network planning, our algorithm can be applied to a subset of cell-edge pixels, to provide fast performance evaluation and thereby suggestions on a proper sub-band division scheme. It is also worth remarking that the algorithm's computing time is largely due to performance evaluation over the

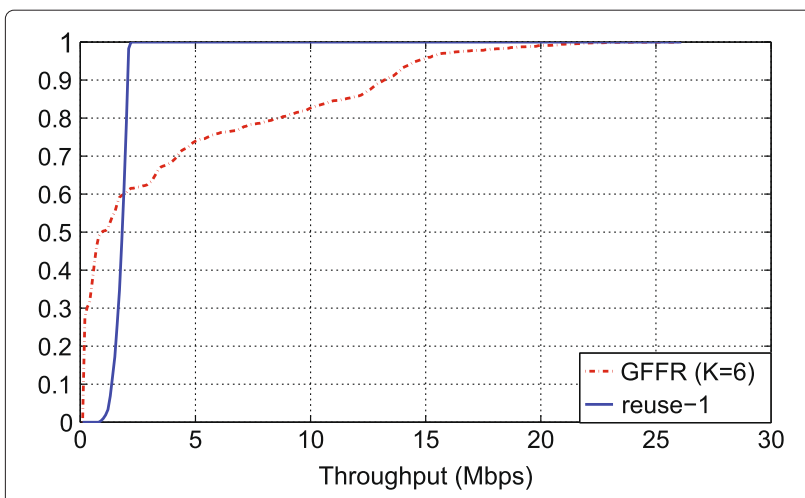

Figure 10 Cell-edge user throughput CDF plot for the Lisbon network. entire area under consideration, for which all pixels are considered. For specific user distributions that typically involve a much smaller number of users than the number of area pixels, the computing time becomes magnitude less.

Figures 10, 11 and 12 provide additional illustration comparing reuse- 1 to GFFR for the Lisbon network with $5 \%$ of the areas being defined as cell edge. The cumulative distribution function (CDF) of cell-edge user throughput is present in Figure 10. The figure shows that about $40 \%$ of the cell-edge users have their throughput significantly improved. These users are the most interference-sensitive ones. Figures 11 and 12 display the cell-edge throughput map for reuse-1 and GFFR with

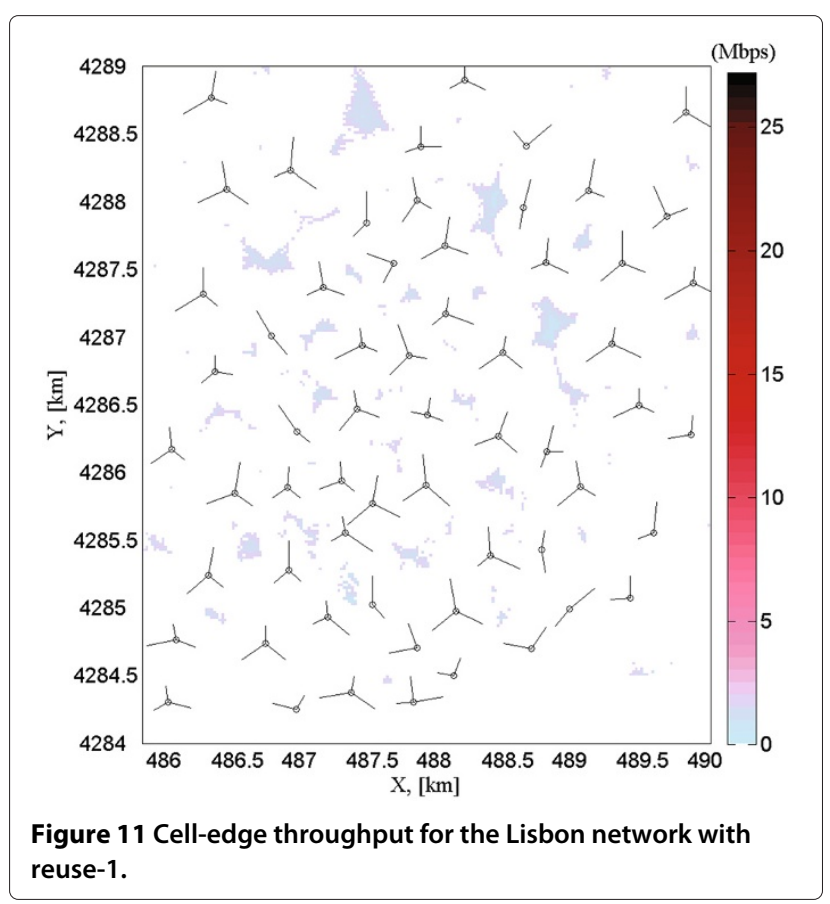




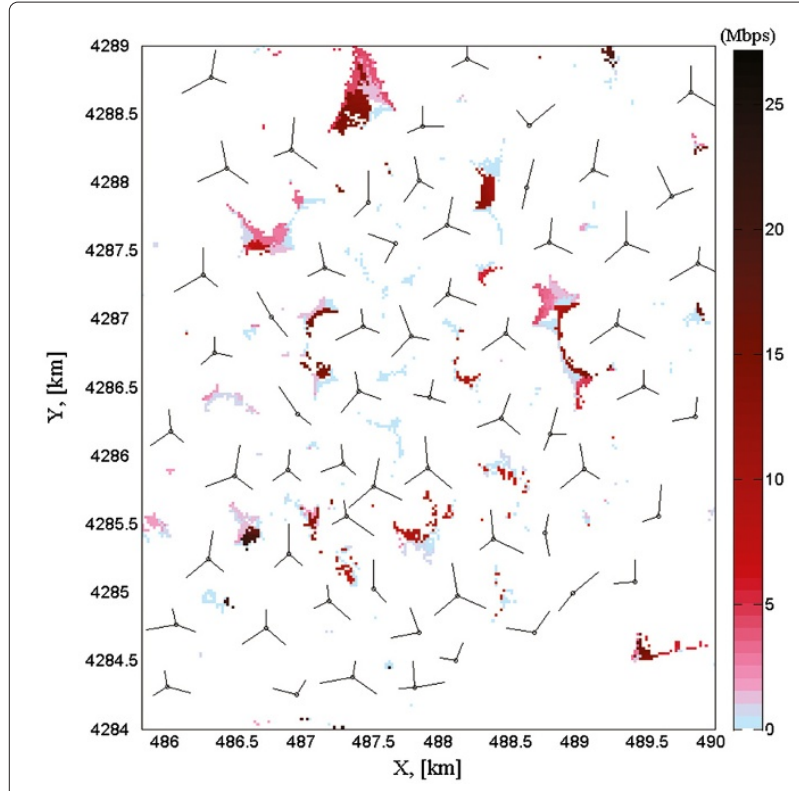

Figure 12 Cell-edge throughput for the Lisbon network with GFFR (K=6).

$K=6$, respectively. It is apparent that a large number of cell-edge pixels achieve significant throughput increase.

\section{Performance trade-off}

Revisiting the results, it is clear that the improvement due to GFFR is strongly related to the proportion of the area that is considered to be cell edge. Low SINR threshold leads to small cell-edge areas with very high interference sensitivity, and therefore more improvement is achieved by GFFR in comparison to reuse-1 for the cell-edge areas. Another factor having a strong influence on performance is the relation between $B_{c}$ and $B_{e}$. In practice, setting the bandwidth values is network-specific, and, to a large extent, the optimal choice depends on the user distribution as well as the performance target. For example, if the cell-edge zones have many users with high qualityof-service requirement, more bandwidth has to be allocated to $B_{e}$. For the results reported in Section "Cell-edge performance", $B_{e}$ corresponds to $60 \%$ of the total bandwidth. The value is quite typical in previous studies of FFR. It results in a throughput loss of $60 \%$ for the cellcenter zones. In general, the performance gain of FFR in the cell-edge zones means a degradation in the cellcenter throughput, as well as lower overall throughput. Similar observations were made in earlier works (e.g., [7]).

For GFFR, enlarging or shrinking the edge bandwidth $B_{e}$ does not change the optimal sub-band allocation. Hence we can easily observe the trade-off between the celledge throughput gain versus the cell-center throughput loss. In Figure 13, we illustrate the cell-center and celledge throughputs for various values of $\frac{B_{c}}{B_{c}+B_{e}}$, for the Berlin network where $5 \%$ of the area is regarded cell edge. The results in the previous section correspond to $\frac{B_{c}}{B_{c}+B_{e}}=40 \%$. In reuse- 1 , the cell-edge and cell-center throughputs are not dependent on the bandwidth partitioning; these throughput values are represented by the two straight lines. As expected, the cell-center throughput as well as the overall throughput improve with $B_{c}$; at the same time, the performance improvement over reuse- 1 in the edge zones goes down gradually. The substantial gain offered by GFFR to cell edge helps in dealing with the performance trade-off between cell center and cell edge. For example, we observe from the figure that, bringing the cell-center throughput loss from $60 \%$ down to $20 \%$, GFFR is still able to deliver throughput values that are about $30 \%$ better than reuse-1 in the cell-edge zones.

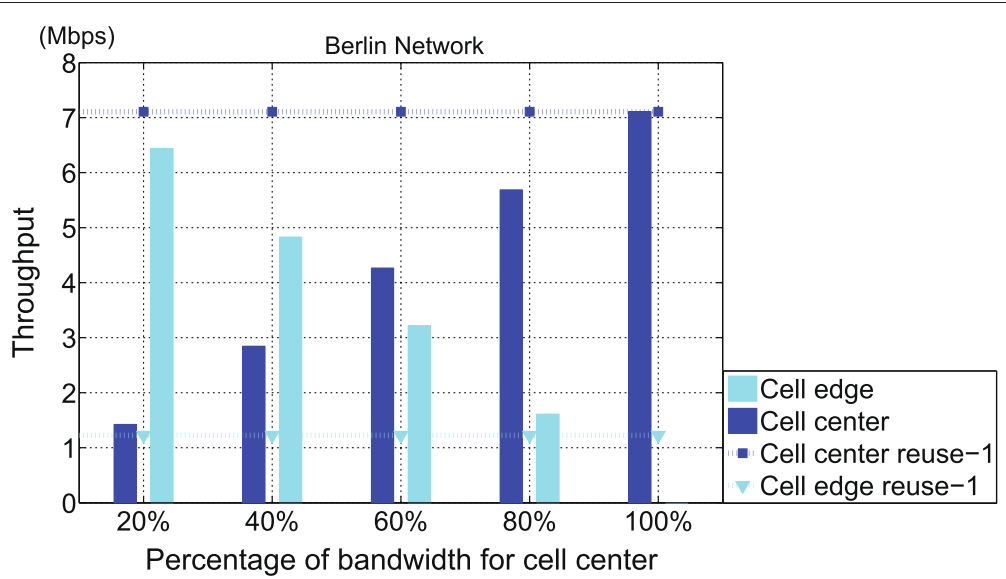

Figure 13 Trade-off between cell-center and cell-edge throughputs for the Berlin network with $5 \%$ cell edge. 


\section{Conclusions}

To overcome the limitations of standard FFR and to address the performance of FFR in large-scale networks with irregular cell structure, we have presented a GFFR scheme that offers higher flexibility in resource allocation. By optimizing sub-band allocation and power assignment, the scheme adapts the utilization of the spectrum and power resource to the level of interference sensitivity of each cell-edge zone. For highly interference-sensitive celledge zones, interference is minimized by sub-band isolation or power reduction, whereas for the other cell-edge zones more bandwidth is allocated if this leads to better performance.

We have studied the complexity of the GFFR optimization problem. A local search algorithm has been developed for problem solution for large-scale networks. Computational experiments show that optimized GFFR delivers substantially higher throughput than reuse-1 at cell edge. In addition, the potential of GFFR, as indicated by the results, is highly useful in dealing with the trade-off between cell-center and cell-edge performance.

By construction, the optimization process in the local search algorithm decomposes by cell. For each cell, the information used in the allocation decision is local (the performance gain and loss of this cell and the neighboring cells). Therefore a promising line of further research is to design distributed implementations of the GFFR scheme. A second topic is to adopt non-uniform power allocation over the sub-bands of each cell. This potentially brings additional performance improvement because it exploits further the diversity over the sub-bands. As the problem complexity grows with this additional dimension of power allocation, it is of particular interest to optimize non-uniform power jointly with the development of distributed and low-complexity algorithms.

Competing interests

Both authors declare that they have no competing interests.

\section{Acknowledgements}

The work has been supported by the ELLIIT Excellence Center and CENIIT, Linköping University, Sweden, and the EU FP7-People-2007-3-1-IAPP-218309 Marie Curie project.

Received: 18 November 2011 Accepted: 30 June 2012 Published: 24 July 2012

\section{References}

1. 3GPP, Evolved universal terrestrial radio access (E-UTRA): physical channels and modulation. Tech. Rep. 36.211, V.8.5.0, 3GPP 2008

2. WIMAX Forum, Mobile WiMAX-part l: a technical overview and performance evaluation. Tech. rep., WIMAX Forum 2006

3. A Simonsson, Frequency reuse and intercell interference co-ordination in E-UTRA. in Proceedings of the 65th IEEE Vehicular Technology Conference (VTC-Spring'07) (Dublin, Ireland, 2007), pp. 3091-3095
4. Ericsson, Inter-cell interference handling for E-UTRA. Tech. rep., 3GPP TSG RAB WG1 Meeting Document, Reference number R1-050764 2005

5. Siemens, Evolved UTRA uplink scheduling and frequency reuse. Tech. rep., 3GPP TSG RAB WG1 Meeting Document, Reference number R1-050476 2005

6. Siemens, Interference mitigation - considerations and results on frequency reuse. Tech. rep., 3GPP TSG RAB WG1 Meeting Document, Reference number R1-050738 2005

7. M Abaii, G Auer, F Bokhari, M Bublin, E Hardouin, O Hrdlicka, G Mange, M Rahman, P Svac, Interference avoidance concepts. Tech. Rep. D.4.7.2 v1.0, IST-4-027756 Project WINNER || 2007

8. SH Ali, VCM Leung, Dynamic frequency allocation in fractional frequency reused OFDMA networks. IEEE Trans. Wirel. Commun. 8, 4286-4295 (2009)

9. G Song, Y Li, Cross-layer optimization for OFDM wireless networks-part I: theoretical framework. IEEE Trans. Wirel. Commun. 4(2), 614-624 (2005)

10. J Jang, KB Lee, Transmit power adaptation for multiuser OFDM systems. IEEE J. Sel. Areas Commun. 21(2), 171-178 (2003)

11. $Y$ Chen, $W$ Wang, $T L i, X Z$ Zhang, $M$ Peng, Fractional frequency reuse in mobile WiMAX. in Proceedings of the 3rd International Conference on Communications and Networking in China (Hangzhou, China, 2008), pp. 276-280

12. H Lei, L Zhang, X Zhang, D Yang, A novel multi-cell OFDMA system structure using fractional frequency reuse. in Proceedings of the 18th IEEE International Symposium on Personal, Indoor and Mobile Radio Communications (PIMRC'07) (Athens, Greece, 2007), pp. 1-5

13. H Luo, Z Zhang, H Jia, G Yu, S Li, Performance comparison of IEEE 802.16e and IEEE 802.20 systems under difference frequency reuse schemes. in Proceedings of the 68th IEEE Vehicular Technology Conference (VTC-Fall'08) (Calgary, Canada, 2008), pp. 1-5

14. YF Zhou, N Zein, Simulation study of fractional frequency reuse for mobile WiMAX. in Proceedings of the 67th IEEE Vehicular Technology Conference (VTC-Spring'08) (Marina Bay, Singapore, 2008), pp. 2592-2595

15. R Giuliano, C Monti, $P$ Loreti, WiMAX fractional frequency reuse for rural environments. IEEE Wirel. Commun. Mag. 15, 60-65 (2008)

16. SE Elayoubi, B Fouretié, On frequency allocation in $3 G$ LTE systems. in Proceedings of the 17th IEEE International Symposium on Personal, Indoor and Mobile Radio Communications (PIMRC'06) (Helsinki, Finland, 2006), pp. $1-5$

17. R Giuliano, P Loreti, F Mazzenga, G Santella, Fractional frequency reuse planning for WiMAX over frequency selective channels. in Proceedings of IEEE International Wireless Communications and Mobile Computing Conference (IWCMC'08) (Crete Island, Greece, 2008), pp. 666-671

18. AL Stolyar, H Viswanathan, Self-organizing dynamic fractional frequency reuse in OFDMA systems. in Proceedings of the 27th Conference on Computer Communications (INFOCOM'08) (Phoenix, USA, 2008), pp. 691-699

19. RY Chang, ZF Tao, JY Zhang, C-CJ Kuo, A graph approach to dynamic fractional frequency reuse (FFR) in multi-cell OFDMA networks. in Proceedings of IEEE International Conference on Communications (ICC'O8) (Beijing, CHINA, 2008), pp. 1-6

20. MC Necker, Local interference coordination in cellular OFDMA networks. in Proceedings of the 66th IEEE Vehicular Technology Conference (VTC-Fall'07) (Baltimore, USA, 2007), pp. 1741-1746

21. MC Necker, Coordinated fractional frequency reuse. in Proceedings of 10th ACM Symposium on Modeling, analysis, and simulation of wireless and mobile systems (Crete Island, Greece, 2007), pp. 296-305

22. T Novlan, JG Andrews, I Sohn, RK Ganti, A Ghosh, Comparison of fractional frequency reuse approaches in the OFDMA cellular downlink. in Proceedings of IEEE Global Telecommunications Conference (GLOBECOM'10) (Miami, USA, 2010), pp. 1-5

23. L Chen, D Yuan, Generalized frequency reuse schemes for OFDMA networks: optimization and comparison. in Proceedings of the 71st IEEE Vehicular Technology Conference (VTC-Spring'10) (Taipei, Taiwan, 2010), pp. $1-5$

24. M Al-Ayyoub, MM Buddhikot, H Gupta, Self-regulating spectrum management: a case of fractional frequency reuse patterns in LTE networks. in Proceedings of IEEE Symposium on New Frontiers in Dynamic Spectrum (Singapore, 2010), pp. 1-12 
25. G Fodor, C Koutsimanis, A Racz, N Reider, A Simonsson, W Müller, Intercel interference coordination in OFDMA networks and in the 3GPP long term evolution system. J. Commun. 4, 445-453 (2009)

26. T Lee, H Kim, J Park, J Shin, Dynamic fractional-frequency reuse for femtocells. in Proceedings of the 5th International Conference on Ubiquitous Information Management and Communication (ICUIMC'11) (Seoul, Korea, 2011), pp. 80:1-80:6, http://doi.acm.org/10.1145/1968613.1968709

27. M Chowdhury, Y Jang, Z Haas, Cost-effective frequency planning for capacity enhancement of femtocellular networks. Wirel. Personal Commun. 60, 83-104 (2011). http://dx.doi.org/10.1007/s11277-0110258-y

28. HC Lee, DC Oh, YH Lee, Mitigation of inter-femtocell interference with adaptive fractional frequency reuse. in Proceedings of IEEE International Conference on Communications (ICC'10) (Cape Town, South Africa, 2010), pp. 1-5

29. T Lee, J Yoon, S Lee, J Shin, Resource allocation analysis in OFDMA femtocells using fractional frequency reuse. in Proceedings of 21 st IEEE International Personal Indoor and Mobile Radio Communications Symposium (PIMRC'10) (Istanbul, Turkey, 2010), pp. 1224-1229

30. M Assaad, Optimal fractional frequency reuse (FFR) in multicellular OFDMA system. in Proceedings of the 68th IEEE Vehicular Technology Conference (VTC-Fall'08) (Calgary, Canada, 2008), pp. 1-5

31. NUL Hassan, M Assaad, Optimal fractional frequency reuse (FFR) and resource allocation in multiuser OFDMA system. in Proceedings of the International Conference on Information and Communication Technologies (ICICT'09) (Cairo, Egypt, 2009), pp. 88-92

32. PC Weeraddana, W Li, M Codreanu, M Latva-aho, Adaptive subcarrier and power allocation for OFDMA systems. in Proceedings of 1st IFIP Wireless Days, (Dubai, UAE, 2008), pp. 1-5

33. KT Kim, Sk Oh, An incremental frequency reuse scheme for an OFDMA cellular system and its performance. in Proceedings of the 67th IEEE Vehicular Technology Conference (VTC-Spring'08) (Marina Bay, Singapore, 2008), pp. 1504-1508

34. Z Xie, B Walke, Enhanced fractional frequency reuse to increase capacity of OFDMA systems. in Proceedings of the 3rd International Conference on New Technologies, Mobility and Security (NTMS'09), (Cairo, Egypt, 2009), pp. 1-5

35. Z Xie, B Walke, Resource allocation and reuse for inter-cell interference mitigation in OFDMA based communication networks. in Proceedings of the 5th Annual ICST Wireless Internet Conference (WICON'10), (Singapore, 2010), pp. 1-6

36. AL Stolyar, H Viswanathan, Self-organizing dynamic fractional frequency reuse for best-effort traffic through distributed inter-cell coordination. in Proceedings of The IEEE Conference on Computer Communications (INFOCOM'09), (Rio de Janeiro, Brazil, 2009), pp. 1287-1295

37. W Fu, Z Tao, J Zhang, DP Agrawal, Clustering based fractional frequency reuse and fair resource allocation in multi-cell networks. in Proceedings of IEEE International Conference on Communications (ICC'10), (Cape Town, South Africa, 2010), pp. 1-5

38. R Ghaffar, R Knopp, Fractional frequency reuse and interference suppression for OFDMA networks. in Proceedings of the 8th International Symposium on Modeling and Optimization in Mobile, Ad Hoc and Wireless Networks (WiOpt'10), (Avignon, France, 2010), pp. 273-277

39. M Rahman, H Yanikomeroglu, Enhancing cell-edge performance: a downlink dynamic interference avoidance scheme with inter-cell coordination. IEEE Trans. Wirel. Commun. 9(4), 1414-1425 (2010)

40. H Xiao, Z Feng, A novel fractional frequency reuse architecture and interference coordination scheme for multi-cell OFDMA networks. in Proceedings of the 71st IEEE Vehicular Technology Conference (VTC-Spring'10), (Taipei, Taiwan, 2010), pp. 1-5

41. RY Chang, ZF Tao, JY Zhang, C-CJ Kuo, Dynamic fractional frequency reuse (D-FFR) for multicell OFDMA networks using a graph framework. Wirel. Commun. Mobile Comput, 1530-8677 (2011)

42. AY Tara, C Hakima, Fractional frequency reuse for hierarchical resource allocation in mobile WiMAX networks. EURASIP J. Wirel. Commun. Netw. 2010, 363065 (2010)

43. L Chen, D Yuan, Beyond conventional fractional frequency reuse for networks with irregular cell layout: An optimization approach and performance evaluation. in Proceedings of the 5 th Annual ICST Wireless Internet Conference (WICON'10), (Singapore, 2010), pp. 1-7
44. K Aardal, CPM van Hoesel, AMCA Koster, C Mannino, A Sassano, Models and solution techniques for the frequency assignment problem. 4OR-Q J. Operat. Res. 1(4), 261-317 (2003)

45. K Aardal, CPM van Hoesel, AMCA Koster, C Mannino, A Sassano, Models and solution techniques for the frequency assignment problem. Ann. Operat. Res. 153, 79-129 (2007)

46. P Björklund, P Värbrand, D Yuan, Optimal frequency planning in mobile networks with frequency hopping. Comput. Operat. Res. 32, 169-186 (2005)

47. STouhami, JM Bourjolly, G Laporte, Optimizing hopping sequences for reducing interference in frequency hopping cellular networks. Eng. Opt. 42,33-44 (2010). http://www.tandfonline.com/doi/abs/10.1080/ 03052150902971690

48. MOMENTUM Project, Data download: public reference scenarios (2005), http://momentum.zib.de/data.php

doi:10.1186/1687-1499-2012-230

Cite this article as: Chen and Yuan: Generalizing and optimizing fractional frequency reuse in broadband cellular radio access networks. EURASIP Journal on Wireless Communications and Networking 2012 2012:230.

\section{Submit your manuscript to a SpringerOpen ${ }^{\circ}$ journal and benefit from:}

- Convenient online submission

- Rigorous peer review

- Immediate publication on acceptance

- Open access: articles freely available online

- High visibility within the field

- Retaining the copyright to your article

Submit your next manuscript at $>$ springeropen.com 